Effect of Single and Combined Altered Auditory Feedback on Stuttering Frequency at Two Speech Rates

\author{
Jennifer MacLeod 1 \\ Joseph Kalinowski $1^{\text {* }}$ \\ Andrew Stuart ${ }^{2}$ \\ and \\ Joy Armson 1 \\ 1School of Human Communication Disorders \\ Dalhousie University \\ 5599 Fenwick St. \\ Halifax, NS, B3H 1R2 \\ Canada \\ 2Department of Psychology \\ Dalhousie University \\ ${ }^{*}$ Corresponding Author, address reprint requests to \\ Internet: KALINOWS@AC.DAL.CA
}

Running head: ALTERED AUDITORY FEEDBACK 


\begin{abstract}
The purpose of the study was to determine if combining delayed auditory feedback (DAF) and frequency altered feedback (FAF) would be more fluency enhancing than either DAF or FAF alone. Ten stutterers read at normal and fast speech rates under nonaltered auditory feedback (NAF), DAF (i.e., a 50 ms delay), FAF (i.e., a one half octave downward shift), and a combination of DAF and FAF [(COMBO), i.e., a $50 \mathrm{~ms}$ delay plus a one half octave downward shift]. Results indicated that stuttering frequency was significantly reduced under all altered auditory conditions at both speech rates relative to the NAF condition. There was, however, no significant differences between the altered auditory feedback conditions (i.e., DAF, FAF, and COMBO). It is suggested that further studies be undertaken to explore the combination of altered auditory feedback conditions, as it may be the case that a floor effect was demonstrated with the singular presentations of DAF and FAF and further improvements in fluency enhancement could not be exhibited in the combined condition. Finally, these findings support the notion that a slowed rate of speech is not necessary for fluency enhancement under conditions of altered auditory feedback.
\end{abstract}




\section{Effect of Single and Combined Altered Auditory Feedback on Stuttering Frequency at Two Speech Rates}

Since the 1950's researchers have extensively examined the role of masking auditory feedback (MAF) and delayed auditory feedback (DAF) in reducing the frequency of stuttering (e.g., Adams \& Hutchinson, 1974; Chase, Sutton, \& Raphin, 1961; Goldiamond, 1962; 1965; Maraist \& Hutton, 1957; Naylor, 1953; Yairi, 1976). After recognizing the fluency enhancing power of both MAF and DAF, some researchers suggested that audition may be an integral source of feedback control in stuttering. That is, researchers hypothesized that because stuttering was influenced by alterations in auditory feedback, its etiology was most likely due to an auditory / perceptual deficit (e.g., Cherry and Sayers, 1956; Mysak, 1966; Webster \& Lubker, 1968). These speculations were criticized by those who suggested that the auditory system was too slow for the on-line correction of speech errors (for a review see Borden, 1979) and those who hypothesized that the alteration in auditory feedback simply created speech motor changes such as slowed speech and/or increasing phonatory duration. For example, Perkins (1979) stated that "In our experience with several hundred stutterers, DAF is effective only as a means of enforcing syllable prolongation. ... In other words, auditory feedback can be manipulated to disrupt fluency, but apparently no one has found a way of manipulating it to improve fluency." (p.102) 
Wingate (1976) hypothesized that the speech of stutterers becomes more fluent under conditions of altered auditory feedback because of an "induced emphasis on phonation, implemented most effectively by an increase in duration " which is expressed through "slowing down" speech (p. 239). The latter notion and derivations of it have prevailed in the field of stuttering for the last twenty years to the extent that investigations of auditory feedback and stuttering have been supplanted by extensive examinations of the speech motor characteristics of stutterers. Many researchers have focused on identifying a deficit which is causal to stuttering by studying the perceptually fluent speech of stutterers (e.g., Armson \& Kalinowski, 1994; Caruso, Abbs, \& Gracco, 1988; Watson \& Alfonso, 1982, 1983, 1987).

Despite the diminished interest in altered auditory feedback over the past 20 years, the role of the auditory system in stuttering has not been completely dismissed. A small number of researchers have either integrated the auditory system into their models of stuttering (e.g., Harrington, 1988; Neilson \& Neilson, 1987, Webster, 1991) or have continued to examine various alterations in auditory feedback to determine which are most efficacious. Recently, Howell, El-Yaniv and Powell (1987) reported a series of experiments in which they compared the ameliorative power of frequency altered feedback [(FAF), in which stutterers' speech was shifted down one octave and fed back to them via earphones], DAF (50 ms), and MAF (produced by an Edinburgh masker). Howell et al. concluded that FAF was more efficacious in the reduction of 
stuttering than either DAF or MAF. These findings have received scant attention. It seems plausible that researchers may have interpreted FAF as another means of auditory feedback which produces fluency by inducing a slow rate of speech.

The hypothesis that a slowed speech rate is necessary for fluency enhancement under conditions of altered auditory feedback was recently examined by Kalinowski, Armson, Roland-Mieszkowski, Stuart, and Gracco (1993). They asked nine stutterers to read at normal and fast speech rates under nonaltered auditory feedback (NAF), MAF, DAF and FAF conditions. Their results showed that similar fluency enhancement occurred under DAF and FAF at both normal and fast speech rates relative to the NAF condition (i.e., between a 70 and $90 \%$ reduction in stuttering frequency). According to the authors, the findings indicated that a slowed speech rate is not necessary for fluency enhancement under altered auditory feedback conditions. They proposed that there are most likely two interdependent factors responsible for fluency enhancement: alteration of auditory feedback and/or modification of speech production.

Following the findings of Howell et al. (1987) and Kalinowski et al. (1993) who found significant fluency enhancement under DAF and FAF conditions, we subsequently hypothesized that combining these two conditions may produce a new more powerful fluency enhancer. Thus, the primary objective of this study was to investigate the effects of auditory feedback alterations in the temporal and frequency characteristics of the speech signal, either alone or in combination, on 
stuttering frequency. Specifically, stuttering frequency was compared under conditions of NAF, DAF, FAF, and a combination of DAF and FAF $(\mathrm{COMBO})$ at both normal and fast rates of speech. It was anticipated that stuttering frequency would decrease at both speech rates under all conditions of altered auditory feedback relative to the NAF condition. Further, the synergistic effect of DAF and FAF in the COMBO condition would be more effective in reducing stuttering than DAF or FAF alone.

Methods

\section{Subjects}

Subjects were ten adult who stutter ranging in age from 21 to 56 years. All subjects were recruited from a Halifax area support group. While none were curretly in therapy, all reported a therapeutic history. Nine of the subjects had normal bilateral hearing sensitivity defined as hearing thresholds of $20 \mathrm{~dB}$ HL (American National Standards Institute, 1989) or better at octave frequencies of 250 to $8000 \mathrm{~Hz}$. One subject presented a mild sensorineural hearing loss on one side and a mild high frequency loss at $4000 \mathrm{~Hz}$ on the other side. All subjects presented with normal bilateral middle ear function (American Speech-LanguageHearing Association, 1990).

Apparatus

All testing was conducted in a double-walled audiometric test suite (Industrial Acoustics Corporation). Subjects sat in a soft backed office chair with a microphone (AKG Model C460B), held by a microphone boom, positioned approximately $15 \mathrm{~cm}$ from their mouth at an orientation 
of $0^{\circ}$ azimuth and $-15^{\circ}$ altitude. Output from the microphone was fed to an audio mixer (Studiomaster Model Session Mix 8-2) and routed to a digital signal processor (Yamaha Model DSP-1) and amplifier (Yamaha Model AX-630) prior to being fed to the subjects' ears through insert earphones (EAR Tone Model 3A). All speech samples were recorded with a video camera (JVC Model S-62U) and a video cassette recorder (JVC Model BR-64004).

During the NAF condition, the speech signal was routed through the digital signal processor unaltered. For the DAF condition, the digital signal processor introduced a delay of $50 \mathrm{~ms}$ to the feedback of the speech signal. In the FAF condition, the frequency of the speech input was shifted down one half octave by the digital signal processor. The specific DAF and FAF setting were chosen because they were found to be effective in a pilot study. For the $\mathrm{COMBO}$ condition, the digital signal processor introduced a delay of $50 \mathrm{~ms}$ and a one half octave downward shift in frequency to the feedback of the speech signal. The amplifier gain for speech input was preset for all conditions of auditory feedback. The output to the earphones was calibrated so a speech signal input to the microphone of $75 \mathrm{~dB}$ SPL had an output in a $2 \mathrm{~cm}^{3}$ coupler of approximately $85 \mathrm{~dB}$ SPL. This calibration procedure attempted to approximate real ear average conversation SPLs of speech outputs from normal hearing talkers. In other words, an attempt was made to provide a speech level output to the speakers' ears consistent with auditory self - 
monitoring during their normal conversation (see Kalinowski et al., 1993 for a detailed description).

Procedures

Subjects were asked to read eight different passages, each slightly longer than 300 syllables which were taken from two junior high school level texts (Sims, G. [1987], Explorers, Creative Teaching Press Inc., and Taylor, C. [1985], Inventions, Creative Teaching Press Inc.). Each passage was read at either a normal or a fast rate of speech under four conditions of auditory feedback: NAF, DAF, FAF, and COMBO. At the fast rate of speech subjects were asked to read as fast as possible while still maintaining intelligibility. Speech rate conditions were counterbalanced across subjects and auditory feedback conditions were randomized for each speech rate. In order to minimize any possible carry-over of fluency enhancement across auditory conditions, subjects produced one to two minutes of monologue under NAF between each reading passage. Subjects were instructed not to use any motor control strategies to reduce or inhibit their stuttering during all experimental conditions. Subjects self-determined "normal" and "fast" speech rates.

Stuttering frequency was determined for the first 300 syllables of each video-taped passage by the first author, a trained speech languagepathology graduate student. Part word repetitions, prolongations, and inaudible postural fixations were identified as instances of stuttering for the calculation of stuttering frequency. Thirty percent of the data set was randomly selected and counted a second time by the same judge. 
Intrajudge agreement for total dysfluencies was 95\%. A second trained research assistant, blinded to the purpose of the study, examined another $30 \%$ of the data and determined interjudge agreement for total dysfluencies was $88 \%$.

Speech rate, in syllables per second, was calculated from the unaltered audio track of the videotape recordings. The analogue speech signal was digitized at a sampling rate of $10 \mathrm{kHz}$ and then displayed on a VAX-4000 workstation using WENDY, a waveform display / analysis program. To determine speaking rate, waveform sections of 50 perceptually fluent syllables which were contiguous and were separated from stuttering episodes by at least one syllable were displayed and analyzed. Durations calculated for the fluent speech samples obtained represented the time between acoustic onset of the first syllable and the acoustic offset of the last fluent syllable, minus pauses that exceeded 100 ms. Most pauses were between 300 and $800 \mathrm{~ms}$ and were typically used by the speakers for an inspiratory gesture. Because most of these pauses had an audible inspiratory record, it is unlikely that they were silent stuttering moments. Fluent speech rate in syllables per second was then determined by dividing the number of syllables in the sample by the duration of each fluent speech sample.

Results

Figure 1 displays means and standard deviations of stuttering frequency as a function of auditory feedback and speech rate condition. As evident in Figure 1, stuttering frequency was substantially reduced 
under all conditions of altered auditory feedback in comparison to the NAF regardless of speech rate condition.

Insert Figure 1 about here

An examination of the stuttering frequency data for linearity, normality and homogeneity of variance revealed positive skewness and unequal variances. As such, prior to inferential statistical analyses, a square root transformation was applied. A two-factor analysis of variance (ANOVA) with repeated measures was performed to investigate the effect of auditory feedback and speech rate condition on stuttering frequency. Results revealed significant main effects of speech rate $[F(1,9)=6.558, p=$ $.0306]$ and auditory feedback condition $[F(3,27)=8.458, p=.0004]$, and a non-significant interaction of speech rate $X$ auditory condition $[F(3,27)=$ $1.130, p=.3546]$. That is, stuttering frequency was significantly higher in the fast rate condition and stuttering frequency was differentially affected under the auditory conditions. A post hoc Student-Newman-Keuls analysis of the main effect of auditory condition revealed all pair-wise comparisons of the altered auditory feedback conditions (i.e., DAF, FAF, and COMBO) with NAF to be significant $(p<.05)$ while all pair-wise comparisons between the altered auditory conditions were nonsignificant $(p>.05)$.

The means and standard deviations of speech rate as a function of auditory feedback and speech rate condition are depicted in Figure 2. As 
some subjects did not produce samples of 50 contiguous fluent syllables, means were calculated from seven, five, and nine values for the NAFnormal speech rate, NAF-fast speech rate, and FAF-fast speech rate conditions respectively. As evident in Figure 2, speech rate increased in the fast rate condition across all auditory feedback conditions.

Insert Figure 2 about here

A two-factor analysis of variance (ANOVA) with repeated measures was performed to investigate the effect of auditory feedback and speech rate condition on speech rate. Results revealed a significant main effect of speech rate condition $[F(1,11)=13.458, p=.0037]$, a nonsignificant main effect of auditory feedback condition $[F(3,29.19)=0.867$, $p=.4692]$ and a non-significant interaction of speech rate $X$ auditory feedback condition $[F(3,19)=3.046, p=.0539]$. In other words, subjects increased their rate of speech when instructed to read at a fast rate, regardless of auditory feedback condition.

Discussion

Two important findings from this study should be noted. First, stutterers experienced significant fluency enhancement under the altered auditory feedback conditions (DAF, FAF and COMBO) relative to the NAF condition at both normal and fast rates of speech. Second, there were no significant differences between the altered auditory feedback conditions (i.e., DAF, FAF, and COMBO). This suggests, contrary to our 
original hypothesis, that the combination of the altered auditory feedback conditions of DAF and FAF employed in this study may not be more fluency enhancing than their singular presentation.

Two possible interpretations can be offered regarding the failed demonstration of an additive effect of DAF and FAF towards fluency enhancement. The first is that no additive effects of altered auditory feedback exist. As such, the fluency enhancement observed during the COMBO condition may be the result of either the DAF or the FAF condition alone. On the other hand, it may be the case that a floor effect was demonstrated with the singular presentations of DAF and FAF and further improvements in fluency enhancement could not be exhibited in the combined condition. By floor effect we are simply saying that there is little room for improvement in fluency. If that is the case then one may entertain the notion that an additive effect does, in fact, exist, however, it could not be revealed in this study.

In order to tease out the possibility of the existence of an additive effect for DAF and FAF, it would be beneficial for subjects not to demonstrate a complete or near complete reduction in stuttering under DAF or FAF alone. This may be achieved by manipulating either subject stuttering severity or the DAF and FAF parameters. With respect to subject severity, it is speculated that severe stutterers who do not display a complete or near complete stuttering reduction under DAF or FAF, have the potential for further additive fluency enhancement under the combined condition. It would be advantageous for future studies 
investigating combined effects of altered auditory alterations to employ subjects who do not demonstrate complete or near complete stuttering reduction under the DAF and FAF. With regard to the auditory parameters, the additive nature may be revealed by examining suboptimal fluency enhancing conditions. It appears that the $50 \mathrm{~ms}$ delay and one half octave downward shift in frequency are optimal or near optimal settings (Hargrave, Armson, Kalinowski, \& Stuart, 1993; Sark, Kalinowski, Armson, \& Stuart, 1993) which result in a complete or near complete reduction in stuttering. Therefore, an additive effect of these acoustic parameters would not be easily discerned. To test the additive nature of these acoustic parameters on stuttering reduction, it may be best to examine settings which result in less than optimal fluency enhancement. For example, if a $25 \mathrm{~ms}$ delay which is not as fluency enhancing as a $50 \mathrm{~ms}$ delay (Sark et al.) and a sub-optimal frequency shift of less than one half octave are used, the potential for revealing the existence of the additive nature of the acoustic parameters may be found.

It should be noted that subjects in this study were able to increase their rate of speech when instructed to do so. Specifically, the mean normal speech rates of subjects in this study ranged from 3.76 to 5.76 syllables/second (s/s) which are comparable to values of 4 to $5 \mathrm{~s} / \mathrm{s}$ found to be characteristic of normal conversational speakers (Netsell, 1981; Pickett, 1980; Walker \& Black, 1950). The same subjects exhibited mean fast speech rates in the range of 4.67 to 7.60 , for the most part exceeding the values cited as representative of a normal speech rate. It is important 
to note that under all altered auditory feedback conditions stutterers showed a substantial reduction in stuttering frequency at both the normal and fast speech rates. These findings support the notion originally put forth by Kalinowski et al. (1993) that a slowed rate of speech is not necessary for fluency enhancement under conditions of altered auditory feedback. In addition, it should also be noted that the auditory feedback conditions did not have a significant effect on speech rate, even the DAF and the COMBO conditions. This suggests that the speech rate reduction normally associated with certain altered auditory feedback conditions may be overcome if subjects are instructed appropriately.

The findings of this study along with those of Howell et al. (1987) and Kalinowski et al. (1993) confirm that alterations in auditory feedback can play an important role in the amelioration of stuttering. As such, further examination of the role of audition in stuttering and of auditory conditions which alter both the temporal and frequency characteristics of the speech signal, either in isolation or in combination, is warranted. In addition, is also suggested that the research into the use of an auditory prosthetic device as an adjunct or an alternative to current stuttering therapy appears justified. Since it has been shown that current stuttering therapies (e.g., rate control therapies) produce speech which is typically perceived to be unnatural sounding to listeners, when compared to nonstutterers' speech or to the stutterers' pre-therapy speech (e.g., Franken, Boves, Peters, \& Webster, 1992; Kalinowski, Noble, Armson \& Stuart, 1994), other means of producing more natural sounding speech 
outside the traditional therapeutic milieu may be more profitable. We suggest that altered auditory feedback should be explored since stuttering has proven to be effectively ameliorated under these conditions and that the perceptually fluent speech produced therein has been evaluated (by both the subjects and the experimenters involved) to be superior in quality to speech produced with current motorically-based therapeutic strategies. This alternative therapeutic approach may be most applicable to those clients who have difficulty producing natural sounding speech and/or those clients who have difficulty in maintaining "motoric" targets. 


\section{References}

Adams, M.R., and Hutchinson, J. (1974). The effect of three levels of auditory masking on selected vocal characteristics and frequency of dysfluency of adult stutterers. Journal of Speech and Hearing Research. 17, 671-675

American National Standards Institute (1989). Specifications for Audiometers (ANSI S3.6 - 1969). New York, NY

American-Speech-Language-Hearing Association (1990). Guidelines for screening for hearing impairments and middle ear disorders. ASHA, 32 (Suppl. 2), 17-24.

Armson, J., \& Kalinowski, J. (1994). Interpreting results of the fluent speech paradigm in stuttering research: Difficulties in separating cause from effect. Journal of Speech and Hearing Research., 37, 69-82.

Borden, G .J. (1979). An interpretation of research on feedback interruption in speech. Brain and Language, 7, 307-319.

Caruso, A.J., Abbs, J.H., \& Gracco, V.L. (1988). Kinematic analysis of multiple movement coordination during speech in stutterers. Brain, $111,439-455$.

Chase, R.A., Sutton, S. \& Rapin, I. (1961). Sensory feedback influences on motor performance. Journal of Auditory Research, 1, 212-223.

Cherry, E., \& Sayers, B. (1956). Experiments upon total inhibition of stammering by external control and some clinical results . Journal of Psychomotor Research, 1, 233-246. 
Franken, C. F., Boves, L, Peter, H.F.M., \& Webster, R.L. (1992). Perceptual evaluation of the speech before and after fluency shaping therapy. Journal of Fluency Disorders, 17, 223-241.

Goldiamond, I. (1962). The maintenance of ongoing fluent behavior and stuttering. Journal of Mathetics, 1, 57-95.

Goldiamond, I. (1965). Stuttering and fluency as manipulatable operant response classes. In Krasner, L. and Ullman, L.P. (Eds.), Research in Behavior Modification. New York: Holt, Rinehart \& Winston.

Harrington, J. (1988). Stuttering, delayed auditory feedback and linguistic rhythm. Journal of Speech and Hearing Research, 31, 36-47.

Howell, P., El-Yaniv, N., \& Powell, D.J. (1987). Factors affecting fluency in stutterers. In H.F.M. Peters and W. Hulstijn (Eds.), Speech Motor Dynamics In Stuttering (pp. 361-369). New York: Springer-Verlag.

Hargrave, S., Armson, J., Kalinowski, J., \& Stuart, A. (1993). Stuttering reduction under frequency-altered feedback at two speech rates. Asha, 35 (10), 142.

Kalinowski, J., Armson, J., Roland-Mieszkowski, M., Stuart, A., \& Gracco, V.L. (1993). Effects of alterations in auditory feedback and speech rate on stuttering frequency. Language and Speech, 36, 1-16.

Kalinowski, J., Noble, S., Armson, J. \& Stuart, A. (1994). Naturalness ratings of the pretreatment and posttreatment speech of adults with mild and severe stuttering. American Journal of Speech-Language Pathology, 3, 65-70. 
Mysak, E. D. (1966). Speech Pathology: Feedback Theory. Springfield, IL: Charles C. Thomas.

Maraist, J.A. \& Hutton, C. (1957) Effects of auditory masking upon the speech of stutterers. Journal of Speech and Hearing Disorders, 22, 385389.

Naylor, R.V. (1953) A comparative study of methods of estimating the severity of stuttering. Journal of Speech and Hearing Disorders, 18, 3037.

Neilson, M. D., \& Neilson, P.D. (1987). Speech motor control and stuttering: A computational model of adaptive sensory-motor processing. Speech Communications, 6, 325-333.

Netsell, R. (1981). The acquisition of speech motor control: A perspective with directions for research. In R. Stark (Ed.), Language Behavior in Infancy and Early Childhood (pp. 127-153). Amsterdam: ElsevierNorth Holland.

Perkins, W. H. (1979). From psychoanalysis to discoordination. In H.H. Gregory (Ed.) Controversies about stuttering therapy (pp. 97-127). Baltimore: University Park Press.

Pickett, J.M. (1980). The Sounds of Speech Communication: A Primer of Acoustic Phonetics and Speech Perception. Baltimore: University Park Press.

Sark, S., Kalinowski, J., Armson, J., \& Stuart, A. (1993). Stuttering amelioration at various feedback delays and speech rates. Asha, 35 (10), 184. 
Starkweather, C.W. (1987). Fluency and Stuttering. Englewood Cliffs, NJ: Prentice Hall.

Walker, C., \& Black, J. (1950) The Intrinsic Intensity of Oral Phrases (Joint Project Report No. 2). Pensacola, FL: Naval Air Station, United States Naval School of Aviation Medicine.

Watson, B.C., \& Alfonso, P.J. (1982). A comparison of LRT and VOT values between stutterers and nonstutterers. Journal of Fluency Disorders, 7, 219-241.

Watson, B.C., \& Alfonso, P.J. (1983). Foreperiod and stuttering severity effects on acoustic laryngeal reaction time. Journal of Fluency Disorders, 8, 183-205.

Watson, B.C., \& Alfonso, P.J. (1987). Physiological bases of acoustic LRT in nonstutterers, mild stutterers, and severe stutterers. Journal of Speech and Hearing Research, 30, 434-447.

Webster, R. L., (1991). Manipulation of vocal tone: Implications for stuttering. In H.F.M. Peters, W. Hulstijn, \& C.W. Starkweather (Eds.), Speech motor control and stuttering. (pp. 535-545). Amsterdam: Elsevier Science Publishers B.V.

Webster, R. L., \& Lubker, B. B. (1968). Interrelationships among fluency producing variables in stuttered speech. Journal of Speech and Hearing Research,, 11,, 754-66.

Wingate, M. E. (1976). Stuttering: Theory and Treatment. New York: Irvington. 
Altered Auditory Feedback

Yairi, E. (1976). Effects of binaural and monaural noise on stuttering. Journal of Auditory Research,, 16, 114-119. 


\section{Acknowledgments}

All authors are considered equal contributors to this paper.

Portions of this paper were presented at the American Speech-LanguageHearing Association Annual Convention, Anaheim, CA, November, 20, 1993. The authors would like to thank Dr. Walter B. Green, Director of the School of Human Communication Disorders, Dalhousie University, for his continued support of our research. The third author is supported by the Medical Research Council of Canada and the Killam Trusts, Dalhousie University. Preparation of this paper was supported in part by NIH Grant DC-00121 awarded to Haskins Laboratories. 


\section{Figure Captions}

Figure 1. Mean values for stuttering frequency as a function of auditory feedback and speech rate conditions $(n=10)$. Error bars represent plus one standard deviation.

Figure 2. Mean values for speech rate (syllables/s) for samples of 50 contiguous fluent syllables as a function of auditory and speech rate conditions $(\mathrm{n}=10)$. Error bars represent plus one standard deviation. (Note: * As some subjects did not produce samples of 50 contiguous fluent syllables, means were calculated from seven, five, and nine values for the NAF-normal speech rate, NAF-fast speech rate, and FAF-fast speech rate conditions respectively.) 